\title{
Real-time science operations to support a lunar polar volatiles rover mission
}

\author{
Jennifer L. Heldmann ${ }^{\mathrm{a}, *}$, Anthony Colaprete ${ }^{\mathrm{a}}$, Richard C. Elphic ${ }^{\mathrm{a}}$, Greg Mattes ${ }^{\mathrm{b}}$, \\ Kimberly Ennico ${ }^{\mathrm{a}}$, Erin Fritzler ${ }^{\mathrm{c}}$, Margarita M. Marinova ${ }^{\mathrm{d}}$, Robert McMurray ${ }^{\mathrm{c}}$, \\ Stephanie Morse $^{\mathrm{c}}$, Ted L. Roush ${ }^{\mathrm{a}}$, Carol R. Stoker ${ }^{\mathrm{a}}$ \\ ${ }^{a}$ NASA Ames Research Center, Division of Space Sciences and Astrobiology, Moffett Field, CA 94035, USA \\ ${ }^{\mathrm{b}}$ NASA Johnson Space Center, Houston, TX 77058, USA \\ ${ }^{\mathrm{c}}$ NASA Ames Research Center, Moffett Field, CA 94035, USA \\ ${ }^{\mathrm{d}}$ BAER Institute, Sonoma, CA 95476, USA
}

Received 17 December 2013; received in revised form 30 June 2014; accepted 26 July 2014

Available online 2 August 2014

\begin{abstract}
Future human exploration of the Moon will likely rely on in situ resource utilization (ISRU) to enable long duration lunar missions. Prior to utilizing ISRU on the Moon, the natural resources (in this case lunar volatiles) must be identified and characterized, and ISRU demonstrated on the lunar surface. To enable future uses of ISRU, NASA and the CSA are developing a lunar rover payload that can (1) locate near subsurface volatiles, (2) excavate and analyze samples of the volatile-bearing regolith, and (3) demonstrate the form, extractability and usefulness of the materials. Such investigations are important both for ISRU purposes and for understanding the scientific nature of these intriguing lunar volatile deposits.

Temperature models and orbital data suggest near surface volatile concentrations may exist at briefly lit lunar polar locations outside persistently shadowed regions. A lunar rover could be remotely operated at some of these locations for the $\sim 2-14$ days of expected sunlight at relatively low cost. Due to the limited operational time available, both science and rover operations decisions must be made in real time, requiring immediate situational awareness, data analysis, and decision support tools. Given these constraints, such a mission requires a new concept of operations.

In this paper we outline the results and lessons learned from an analog field campaign in July 2012 which tested operations for a lunar polar rover concept. A rover was operated in the analog environment of Hawaii by an off-site Flight Control Center, a rover navigation center in Canada, a Science Backroom at NASA Ames Research Center in California, and support teams at NASA Johnson Space Center in Texas and NASA Kennedy Space Center in Florida. We find that this type of mission requires highly efficient, real time, remotely operated rover operations to enable low cost, scientifically relevant exploration of the distribution and nature of lunar polar volatiles. The field demonstration illustrated the need for science operations personnel in constant communications with the flight mission operators and the Science Backroom to provide immediate and continual science support and validation throughout the mission. Specific data analysis tools are also required to enable immediate data monitoring, visualization, and decision making. The field campaign demonstrated that this novel methodology of real-time science operations is possible and applicable to providing important new insights regarding lunar polar volatiles for both science and exploration.
\end{abstract}

Published by Elsevier Ltd. on behalf of COSPAR.

Keywords: Moon; Volatiles; Rover; Missions

\footnotetext{
* Corresponding author. Tel.: +1 650604 5530; fax: +1 6506046779

E-mail address: jennifer.heldmann@nasa.gov (J.L. Heldmann).
} 


\section{Introduction}

\subsection{Lunar polar rover mission concept}

Understanding the form, distribution, and content of water/ice and other volatiles at the lunar poles can have a significant impact on our scientific understanding of the Moon and on plans for utilizing the resources on the Moon for sustained human exploration. Recent orbital remote sensing and surface impact data from lunar spacecraft have provided information on the potential presence and distribution of water/ice on the Moon (Pieters et al., 2009; Sunshine et al., 2009; Colaprete et al., 2010). However, these datasets provide only an initial understanding of the form and distribution of lunar volatiles. Ground-truthing at higher resolution on the lunar surface is now required to further our understanding of volatile form and distribution on the Moon (Sanders et al., 2012).

To further characterize lunar polar volatile deposits at smaller scales than has been possible to date and to assess ISRU potential of such deposits, the National Aeronautics and Space Administration (NASA) and the Canadian Space Agency (CSA) are developing a lunar polar rover mission concept to prospect for lunar volatiles and demonstrate in situ resource utilization (ISRU) on the lunar surface (Sanders and Larson, in this issue). This mission has the goals of (1) locating near subsurface volatiles, (2) excavating and analyzing samples of the volatile-bearing regolith, and (3) demonstrating the form, extractability and usefulness of the materials. The mission is a rover-based platform that includes neutron and near infrared spectrometers to prospect for hydrogen sources and volatiles, a drilling system to collect samples down to one meter below the surface, and a sample analysis oven with a gas chromatograph/mass spectrometer to heat and analyze water and other volatiles released from subsurface samples.

This mission is unique in that mission operations will be conducted in real time, thereby necessitating a new concepts of operations. Since the rover will be operated during a time period of $\sim 2-14$ days of sunlight near a lunar pole and the results of the two prospecting instruments (neutron and near infrared spectrometers) will not be known a priori, the Science Team will need to make real-time decisions based on this data. For example, the science team will need to identify the locations of hydrogen (and volatile) hotspots as well as decide when and where to auger and/or drill to collect samples for the sample analysis oven and ISRU measurements. The real time cadence of this mission thereby necessitates that the mission employ real time science operations to achieve the stated mission goals.

\subsection{Site selection}

Site selection is a key driver of the lunar polar rover mission duration and hence of the mission concept of operations. Site selection is predicated on identifying a region(s) with (1) high hydrogen concentrations, (2) ice stability, (3) visibility from Earth (so a relay satellite is not required to perform the mission), and (4) brief periods of sunlight to allow solar power to be utilized. The need for high hydrogen concentrations drives the mission to the lunar north or south polar regions. Preliminary analysis has also shown that these regions typically experience continual visibility from Earth which enables DTE (direct to Earth) communications without a relay satellite (Heldmann et al., 2012). We now examine the final two criterion (ice stability and brief periods of sunlight) to further refine the site characteristics that are key to defining the science operations constraints.

Recent work has shown that stable ice may exist outside of permanent shadow near the lunar poles. Thermal modeling coupled with Diviner lunar radiometer measurements from the Lunar Reconnaissance Orbiter (LRO) has shown that cryogenic temperatures exist outside of permanent shadow in near-surface regions (Paige et al., 2010). Fig. 1 shows a map of depth to stable ice for the lunar south polar region. Areas where the depth to stable ice is zero meter (white regions in Fig. 1) are primarily regions of permanent shadow. All other regions with a range of depths greater than zero and up to one meter are areas that receive on the order of several days of sunlight per month. The low sun angle coupled with the relative short duration of solar illumination results in the cryogenic subsurface temperatures which enable cold-trapping of water ice and other volatiles, even outside of permanently shadowed regions.

Fig. 2 shows the maximum days of expected sunlight based on Lunar Orbiter Laser Altimeter (LOLA) data from LRO. Areas of permanent shadow (purple areas in Fig. 1 representing zero days of sunlight) correspond to areas where ice is stable on the surface. However, the confluence of Figs. 1 and 2 shows that there are ample regions where ice is stable within the upper meter of the lunar surface

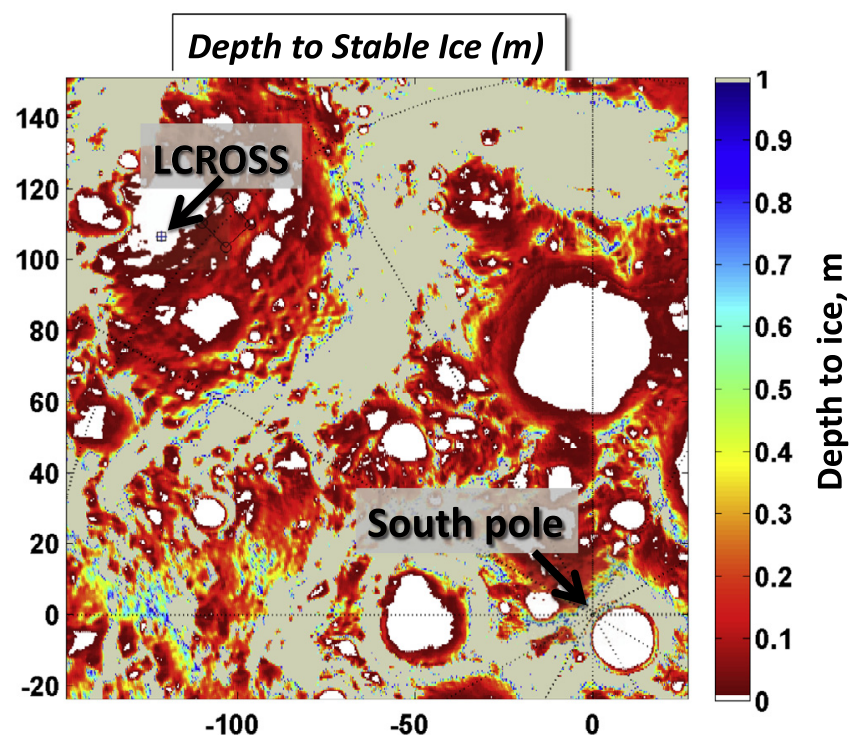

Fig. 1. Polar stereographic map projection of the south pole region of the Moon indicating depth to stable ice. Axes are labeled in kilometers with the map origin at the south pole. The location of the lunar south pole and LCROSS impact site are indicated by black arrows. 


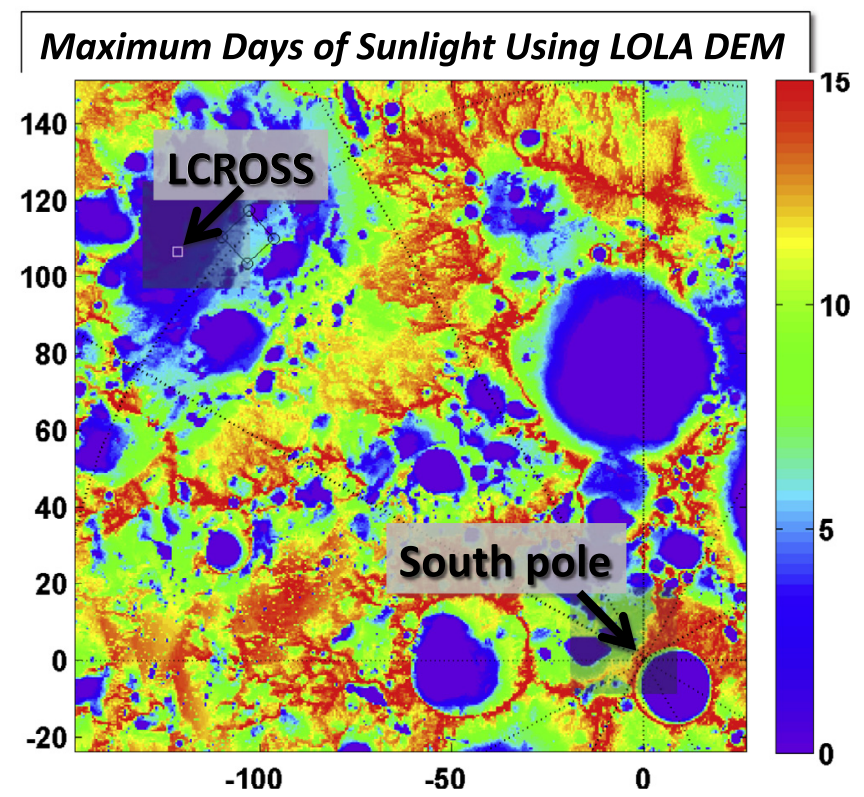

Fig. 2. Polar stereographic map projection of the south pole region of the Moon indicating the maximum days of sunlight based on LOLA digital elevation model data. Axes are labeled in kilometers with the map origin at the south pole. The location of the lunar south pole and LCROSS impact site are indicated by black arrows.

which enables relative ease of access to the volatiles, coupled with multiple days of sunlight (actual number of days depends on location but generally varies from several days to less than two weeks, Fig. 2). Solar illumination enables relative simplicity in rover surface operations in terms of rover navigation and provides more benign operating temperatures on the lunar surface. The lunar polar rover concept is designed to operate only during daylight in order to reduce complexity and cost with respect to both engineering and operations.

The limited duration of sunlight in regions where ice is expected to be stable in the upper one meter of the near surface is the main driver for the lunar polar rover mission duration. Since the mission is not designed to survive the lunar night, all surface operations must be completed in the $\sim 2-14$ days of available sunlight (the exact duration of sunlight depends on the precise landing site, Fig. 2). Due to this limited operational time on the lunar surface, all science operations must be near real time. This requirement demands immediate situational awareness plus realtime data analysis and decision support tools. Hence site selection affects the concept of operations and drives the need for efficient real time science operations.

\section{Hawaii 2012 field campaign}

\subsection{Overview}

In July 2012 a full-scale field demonstration for testing of both technologies required to enable a lunar polar rover mission and concepts of operations was conducted. With help from the Pacific International Space Center for
Exploration Systems (PISCES), a lunar rover prototype (provided by the Canadian Space Agency) was equipped with a suite of prospecting instruments (neutron spectrometer and near-infrared spectrometer) and volatile characterization instruments (drill and auger for subsurface sample collection plus the ISRU-specific instruments LAVA (Lunar Advanced Volatile Analysis) and OVEN (Oxygen and Volatile Extraction Node)). The rover was operated at a lunar analog site on the upper slopes of Mauna Kea, Hawaii with a mission operations center co-located in Hawaii, rover navigation center in Canada, a Science Backroom at NASA Ames Research Center in California, and support teams at NASA Johnson Space Center in Texas and NASA Kennedy Space Center in Florida.

A general concept of operations was developed prior to the field deployment to maximize success and achieve mission goals. The near-infrared spectrometer and neutron spectrometer are designed to prospect for surface and subsurface volatiles, respectively, while the rover is driving across the terrain. The spectrometer data is used by the Science Team to not only map the distribution of volatiles but also to make real-time decisions regarding rover operations. Such decisions include the time(s) and location(s) to stop and drill and/or auger as well as process samples using the LAVA and/or OVEN payload elements.

\subsection{Console architecture}

The analog field campaign was intentionally designed with a distributed mission operations architecture which is likely most representative of the console position distribution for the actual lunar mission. The 2012 field campaign console architecture is shown in Fig. 3 where science-specific positions are highlighted in yellow. The main Flight Control Center was located in Hawaii although was geographically separated from the rover field site in order to maintain a high fidelity to the lunar mission scenario. The support teams based at NASA Ames Research Center, NASA Johnson Space Center, NASA Kennedy Space Center, and the Canadian Space Agency were all connected to the main Flight Control Center via both voice and online electronic systems.

In Hawaii, several console positions within the flight mission operations hierarchy reflected the need for timely science decision-making including an overall Science Lead, a Real-Time Science Lead, and a Spectrometer Lead. The Science Lead was responsible for directly communicating science-related information between the Flight Director and a Science Backroom. The Real-Time Science console position was responsible for recommending rover operations modifications based on the spectrometer prospecting data. Rover plan modifications were typically reached via consensus among the Science Team where the Science Team consisted of the Science Backroom plus the Science Lead, Real-time Science Lead, and Spectrometer Lead. Examples of such modifications included, but were not limited to, requesting a rover to stop when traversing a 


\section{Console Architecture}

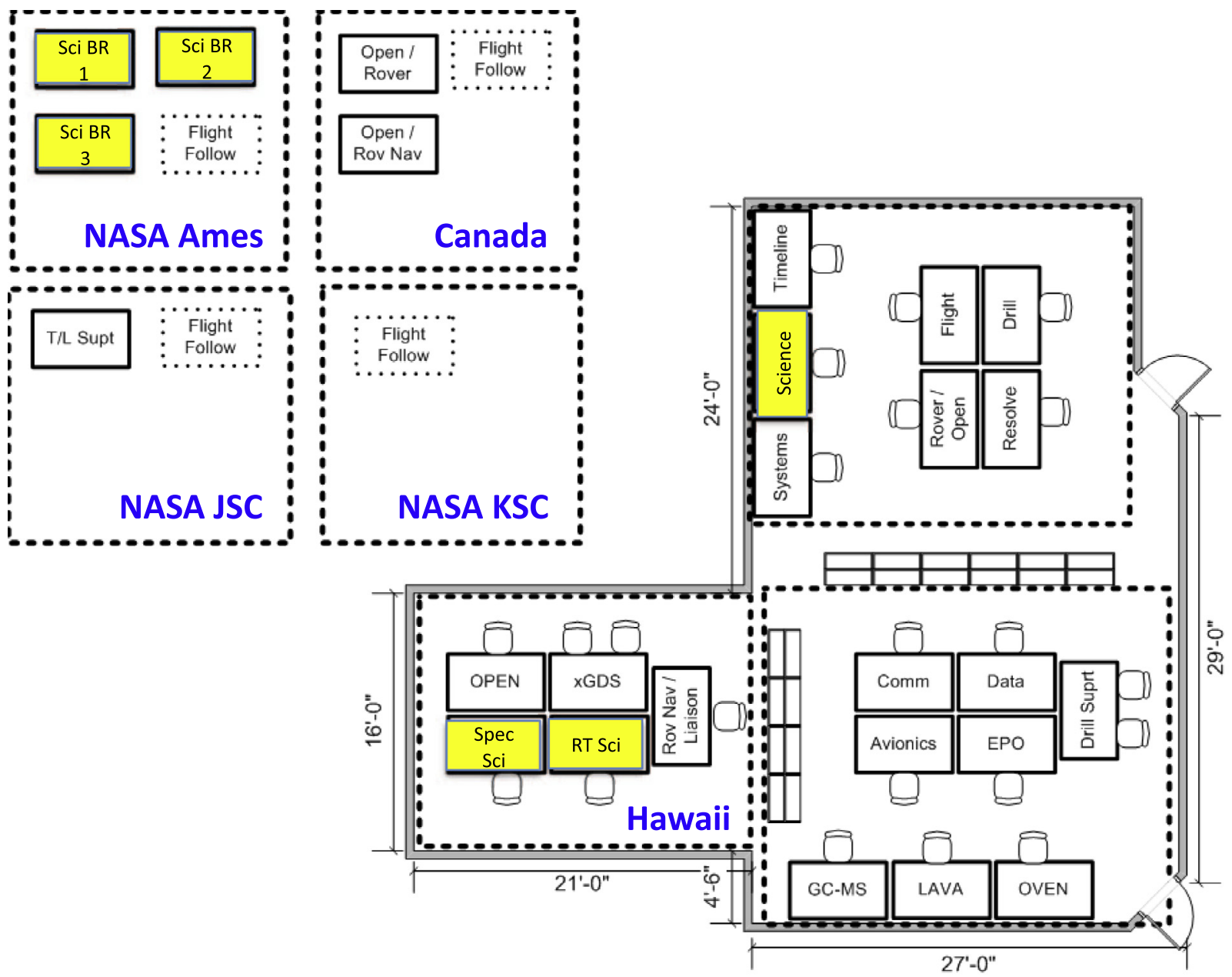

Fig. 3. The console architecture for the 2012 lunar rover field campaign. Science positions are highlighted in yellow (Sci BR $=$ Science Backroom, Spec Sci $=$ Spectrometer Lead, RT Sci $=$ Real-time Science Lead, Science $=$ Science Lead). The physical location of each console center is listed in blue. $($ For interpretation of the references to color in this figure legend, the reader is referred to the web version of this article.)

high volatile content area, a hydrogen hotspot localization procedure to map volatile distribution at high resolution, a drill and/or auger procedure to sample the subsurface, or a rover speed adjustment based on spectrometer readings. The Real Time Science position was also tasked with answering questions about science posed by the Flight Director and assisting with monitoring the real-time spectrometer data. The Spectrometer Lead was also located in the Flight Control Center to support monitoring of the neutron and near-infrared spectrometers which provided data on the volatile distributions of the surface and subsurface (up to $\sim 1 \mathrm{~m}$ ), respectively. This data was used to identify regions of high hydrogen and volatile content for further vertical and horizontal volatile mapping and also to identify optimal target areas for more in-depth volatile characterizations with the drill/auger and LAVA/ OVEN systems.

Supporting these Flight Control Center console positions was a Science Backroom that was tasked with monitoring the data, conducting in-depth data analysis to support mission decision-making, and conducting any rover traverse replanning as required based on the data and information obtained from prior roving activities (Fig. 4). Specific positions within the Science Backroom included a Stenographer, Traverse Planning Lead, and a Communications Lead. The Stenographer was responsible for documenting science activities plus science-related decision making processes and outcomes. This person closely monitored and documented all science-related events for future use in improving data analysis and understanding and to provide a record of science activities throughout the mission. The Traverse Planning Lead was responsible for updating traverse paths based on the Science Team inputs and recommendations based on the rover payload data. The Traverse Planning Lead uploaded revised traverse plans which were then sent to the rover by the Flight Control Center for immediate implementation. The Communications Lead was responsible for providing 


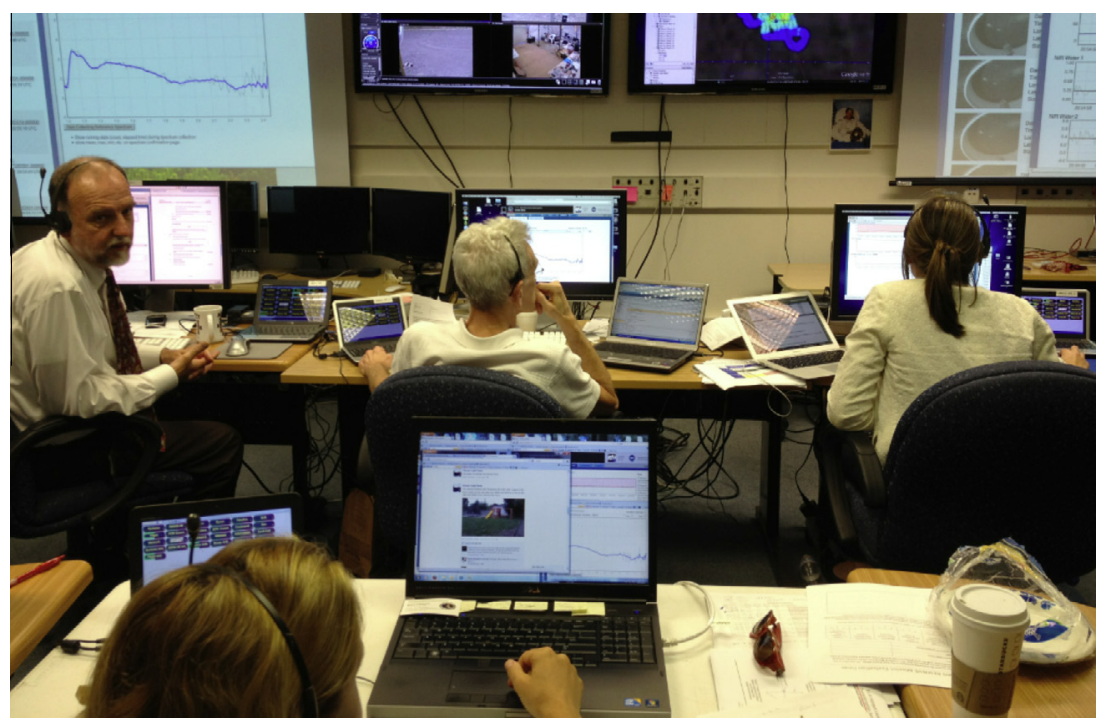

Fig. 4. Image of the Science Backroom at NASA Ames Research Center during the 2012 lunar rover field campaign.

two-way communication between the Science Backroom and the science positions in the Flight Control Center. In addition to these three positions, several more Science Team members participated in the Science Backroom to provide additional science support during the mission. These scientists focused on more in-depth data analysis from the prospecting and ISRU payload data to provide more detailed cross-correlations of datasets and provide a more thorough understanding of the volatile distributions. These team members also focused on more long-term strategic planning for the mission based on information gleaned from the previously collected datasets and compiled summaries of science activities at the end of each shift.

\subsection{Communications}

Strict communications protocols were invoked to ensure efficient and effective communication in real-time during the field demonstration. For example, the Science Backroom at NASA Ames conversed with the Spectrometer Lead and Real-Time Science position (located in the Flight Control Center in Hawaii) on a dedicated voice loop. The Spectrometer Lead and the Real-Time Science position conversed with the overall Science Lead (to alleviate confusion, the Science Backroom did not communicate directly with the Science Lead in the Flight Control Center), and the Science Lead relayed all science-related operational information to the Flight Director responsible for the overall mission. The Science Lead was also responsible for relaying science-related operations questions from the Flight Director back through the science support structure (Spectrometer Lead, Real-Time Science, and Science Backroom) to solicit science recommendations and/or clarifications when necessary. Fig. 5 shows a diagram of the communications flow during the field test.

Communications were enabled by both voice and electronic communications capabilities (email and chat

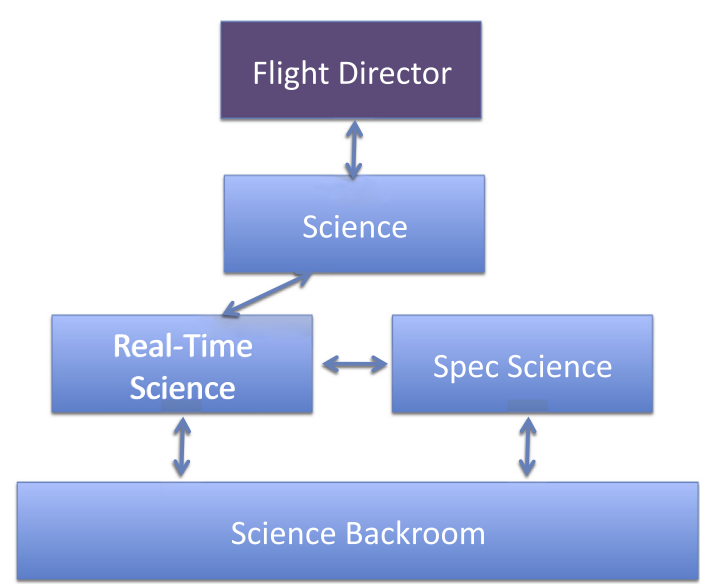

Fig. 5. The science communications hierarchy for the 2012 field campaign. Arrows indicate directions of permissible communication among console positions.

functions). Voice communications were enabled on dedicated voice loops where communications were only allowed as described above. Such a structure ensured that each console position received only the information needed and was not confused by cross-talk from parties unrelated to that particular console position's duties. Additional communications were enabled by email transmissions amongst team members which proved useful for longer discussions and/or when electronic files needed to be shared amongst team members. For more rapid and short electronic communications, each team member was equipped with a chat program which allowed real-time communication in a text only format.

\subsection{Real time decision making}

The nature of the lunar polar rover mission as tested in the Hawaii field campaign during July 2012 is such that the 
rover operations is dictated in real time by incoming prospecting data to identify areas of interest for more detailed study. The near infrared spectrometer is suited for measuring surface volatiles while the neutron spectrometer is specialized at measuring the volumetric content of hydrogen (and hence volatiles) in the near subsurface.

Prior to the field campaign, the Science Team developed criterion upon which rover operations decisions would be based dependent upon the spectrometer prospecting data. These decision making criterion are summarized in Fig. 6. If both the near infrared and neutron spectrometers produced a positive signal for volatiles, the Science Team would recommend that the rover stop in its traverse and auger and/or core to sample the subsurface. If the near infrared signal was negative (no surface volatiles) but the neutron spectrometer signal was positive (indicative of volumetric hydrogen), then the Science Team would recommend to auger in this location. If the neutron spectrometer signal was negative while the near infrared signal was positive, the Science Team would recommend to auger and/or core only if time and rover energy levels permit. If both the near infrared and neutron signatures were negative then the rover should continue its traverse unless the mission is less than $12 \mathrm{~h}$ from completion and there have been no subsurface samples yet acquired. In this case the Science Team would recommend to auger. In all cases, an area of interest mapping procedure was conducted prior to any auger or coring activities. The area of interest mapping consisted of driving the rover on a predetermined path to map out the volatiles in a specific region. This information was then used to identify the precise location of highest volatile content in order to determine the optimal location for auger and/or core activities.

\subsection{Ground support software}

Real-time science operations activities require novel software capabilities. Specifically, the software must be

\begin{tabular}{|l|l|l|}
\multicolumn{1}{c|}{$\begin{array}{l}\text { Subsystem } \\
\text { Alert Status }\end{array}$} & \multicolumn{1}{c|}{ NIR Signal: YES } & \multicolumn{1}{c|}{ NIR Signal: NO } \\
$\begin{array}{r}\text { NS Signal: } \\
\text { YES }\end{array}$ & $\begin{array}{l}\text { Indication: Both } \\
\text { volumetric and surficial } \\
\text { volatiles present. }\end{array}$ & $\begin{array}{l}\text { Indication: Subsurface } \\
\text { volatiles only. }\end{array}$ \\
\cline { 2 - 4 } & $\begin{array}{l}\text { Recommendation: } \\
\text { Auger and/or core }\end{array}$ & $\begin{array}{l}\text { Recommendation: } \\
\text { Auger }\end{array}$ \\
\hline \multirow{4}{*}{$\begin{aligned} \text { NS Signal: } \\
\text { NO }\end{aligned}$} & $\begin{array}{l}\text { Surficial material only, } \\
\text { or volumetrically minor } \\
\text { constituent (<1 wt\% } \\
\text { equivalent) }\end{array}$ & $\begin{array}{l}\text { No volatiles of } \\
\text { significance. }\end{array}$ \\
\cline { 2 - 3 } & $\begin{array}{l}\text { Recommendation: } \\
\text { Drill/auger if time/ } \\
\text { energy permits }\end{array}$ & $\begin{array}{l}\text { Recommendation: Only } \\
\text { auger if the 12-hr flight } \\
\text { rule is in play. }\end{array}$ \\
\hline
\end{tabular}

Fig. 6. Spectrometer truth table indicating recommended rover operations based on near-infrared spectrometer (NIR) and neutron spectrometer (NS) signals. able to support (1) real-time monitoring of the prospecting data to support science decision-making and (2) real-time traverse plan updates. This field campaign utilized customized exploration ground data system software (xGDS) to serve these purposes. xGDS is discussed briefly here in the context of science operations.

xGDS served multiple functions to enable the science operations of this rover mission. One main use of xGDS was for rover traverse planning. The Science Team developed rover traverse plans a priori based on available satellite imagery. A sample traverse plan is shown in Fig. 7. Here the green and orange lines represent nominal paths to be executed by the rover based on sites of interest identified by the Science Team (white arrows) while also taking into account engineering inputs such as slopes and communications coverage determined by the engineering team which dictate the regions where the rover is allowed to enter. (Slope and communications maps were available as overlays in xGDS to assist with traverse planning activities). The Traverse Planning Lead within the Science Backroom also updated rover traverse plans during the mission based on incoming science data. xGDS enabled the Science Team to replan rover traverses and activities based on science data and upload these plans for immediate rover execution.

xGDS was also used for monitoring rover activities. Rover traverse plans and the paths actually executed by the rover were continually updated and superimposed on a satellite image for context in real time. This function allowed the Science Team to continually monitor the location of the rover as well as understand the instrument data as a function of geographic location.

To assist with real-time prospecting data monitoring, several tools were developed within xGDS. Specialized data displays were developed to monitor rover activities correlated with science data. For example, data displays included color-coded paths following the rover traverse which indicated relative amounts of volatile abundances with respect to geographic location. Fig. 8 shows a map of neutron counts from the field test as a function of position. The colored regions represent variations in neutron counts (and hence hydrogen abundance). xGDS also displayed continually updated strip charts plotting the instrument data as a function of time (Fig. 9). Neutron data was displayed as neutron counts (directly proportional to hydrogen content). For the near infrared data, the raw spectra were monitored and band depth algorithms were employed to automatically calculate and display the strength of several bands for rapid assessment of water content. Fig. 9 shows neutron counts (top chart) and near infrared water band depth (bottom chart) as a function of time during the field campaign. Readings above a pre-calibrated threshold for the neutron spectrometer (red region in Fig. 9) are indicative of volumetric hydrogen while peaks in the near infrared band depth chart are indicative of surface water. Fig. 9 shows examples of elevated neutrons (bulk water only), elevated near 


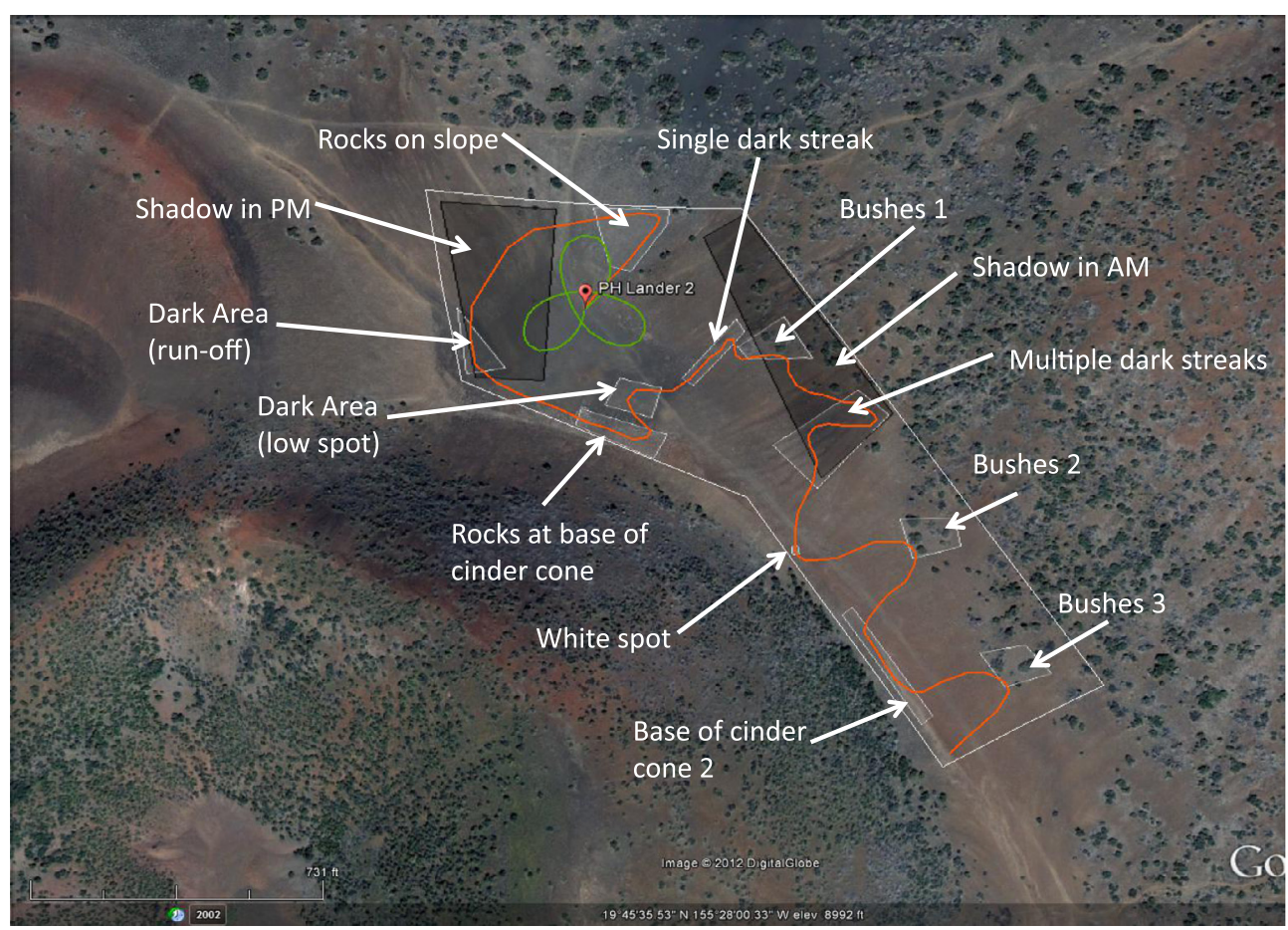

Fig. 7. Sample traverse path developed by the Science Team prior to the Hawaii field deployment. Recommended rover traverses are shown in green and orange. Areas of interest identified using the remote sensing imagery are indicated in white. (For interpretation of the references to color in this figure legend, the reader is referred to the web version of this article.)

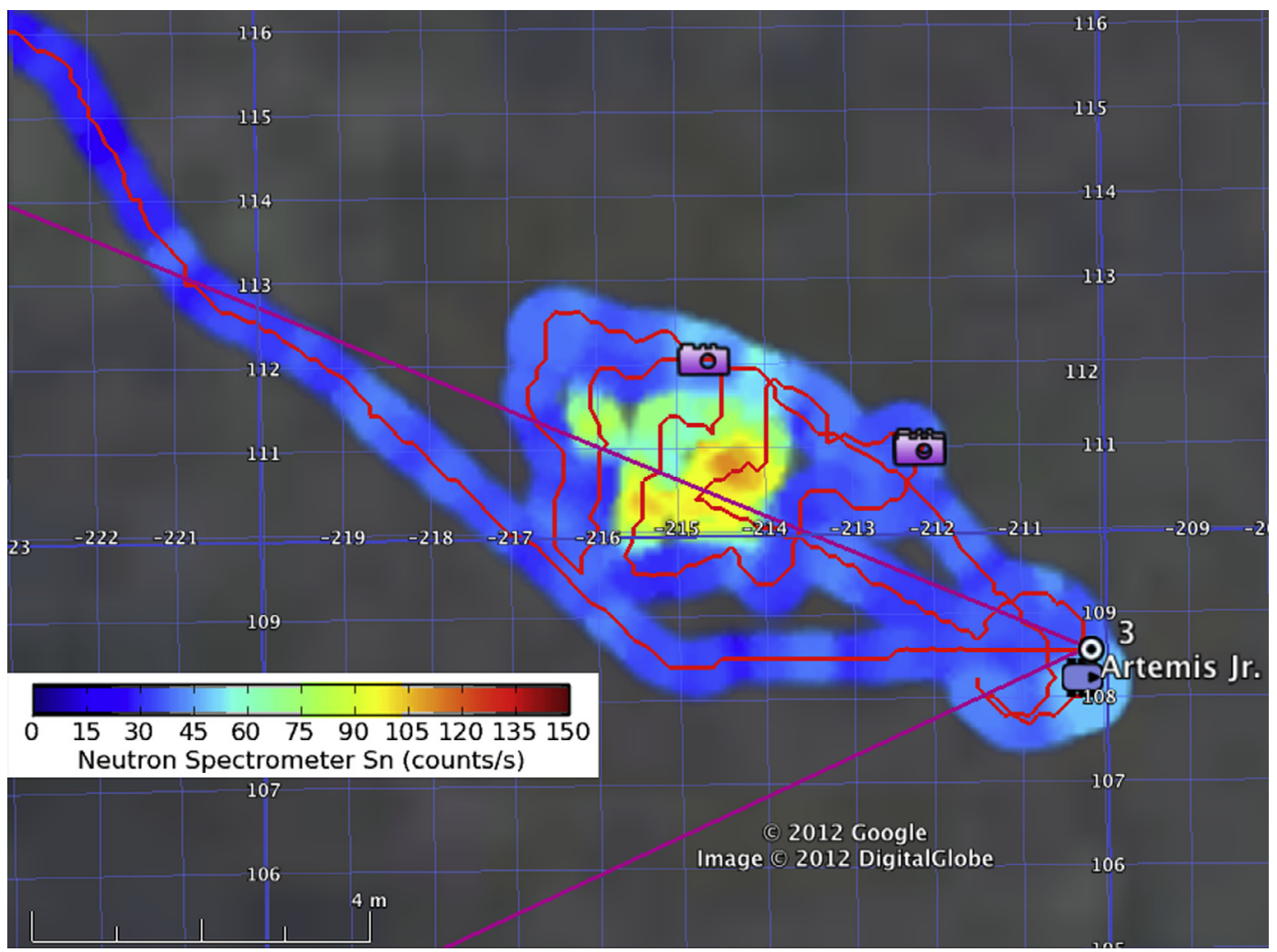

Fig. 8. Georeferenced map created in the exploration ground data system (xGDS) showing neutron spectrometer counts as a function of location. The map was continually updated in real-time during rover operations. 


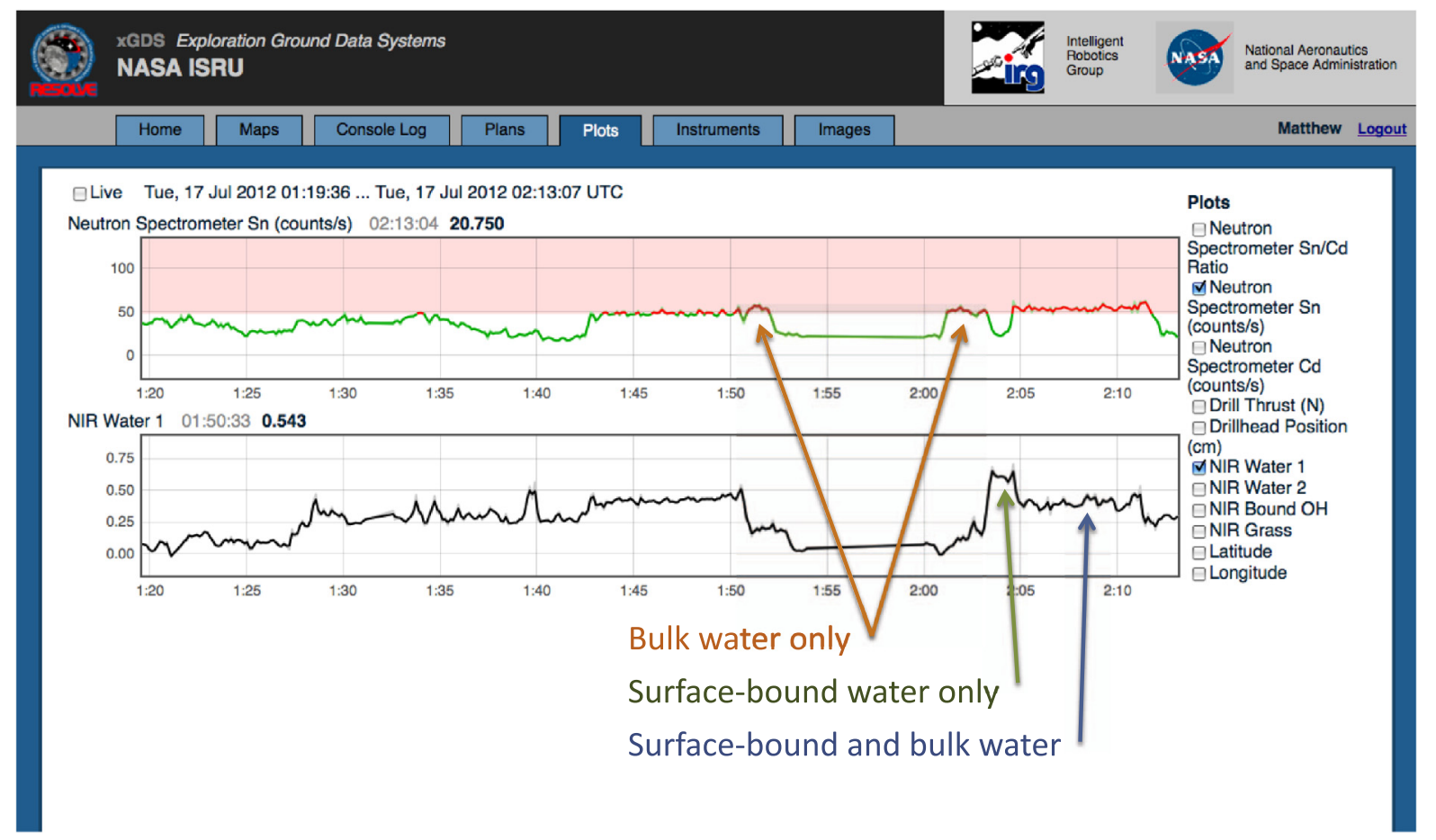

Fig. 9. Sample strip chart from the 2012 field campaign showing neutron spectrometer counts (top panel) and near-infrared spectrometer (NIR) water band depth as a function of time. Neutron counts above a predetermined threshold (red region in top panel) are indicative of bulk water. Surface bound water is detected by increases in the near-infrared band depth. (For interpretation of the references to color in this figure legend, the reader is referred to the web version of this article.)

infrared band depth (surface bound water only), and an instance of elevated neutron counts and water band depth (bulk and surface bound water). A second example demonstrating the utility of the data strip charts is shown in Fig. 10 where drill activities are represented. Here the drill engineering data is used to monitor drilling behavior. Initial drill contact with the ground is measured when the drill thrust (Fig. 10, top panel) registers a slight blip. Following ground contact the drill thrust remains low with time (Fig. 10, top panel) while the drill head position increases (Fig. 10, middle panel), indicating that the drill was penetrating into the subsurface through relatively soft material. A layer of harder material was encountered when the drill thrust increased and spiked significantly while the drill position remained relatively constant. The drill was then extracted from the ground as the drill thrust returned to the baseline level of zero Newtons and the drill position decreased, indicating the drill was being pulled up from the subsurface. Throughout this drilling process, no appreciable water signature was observed with the near infrared spectrometer (Fig. 10, bottom panel), indicating that water was not present in the subsurface at this location. This example shows the utility of multiple datasets displayed and monitored in a time series to understand rover subsystem functions as well as to assess the science implications of the mission activities.

\section{Lessons learned}

The 2012 ISRU field campaign provided a unique opportunity to test science operations in a high fidelity environment. A value of analog field testing is the ability to learn about concepts of operations in a low-cost, lowrisk environment and utilize lessons learned to increase the robustness and optimize the mission concept design prior to flight. Here we review some of the key lessons learned to be incorporated into future planning and mission implementation efforts for both analog field campaigns and lunar flight opportunities.

\subsection{Console architecture}

The general console architecture employed during the 2012 field campaign was efficient and effective for achieving mission goals. A key lesson was the importance of having science interests adequately represented in the Flight Control Center. This was accomplished by having a Science Lead in direct communication with the Flight Director as well as having the Real-Time Science Lead and Spectrometer Lead included in the operations plan to ensure that science considerations are a key component driving the mission operations.

The Science Backroom proved most useful for support purposes and not for real-time decision making. The main 


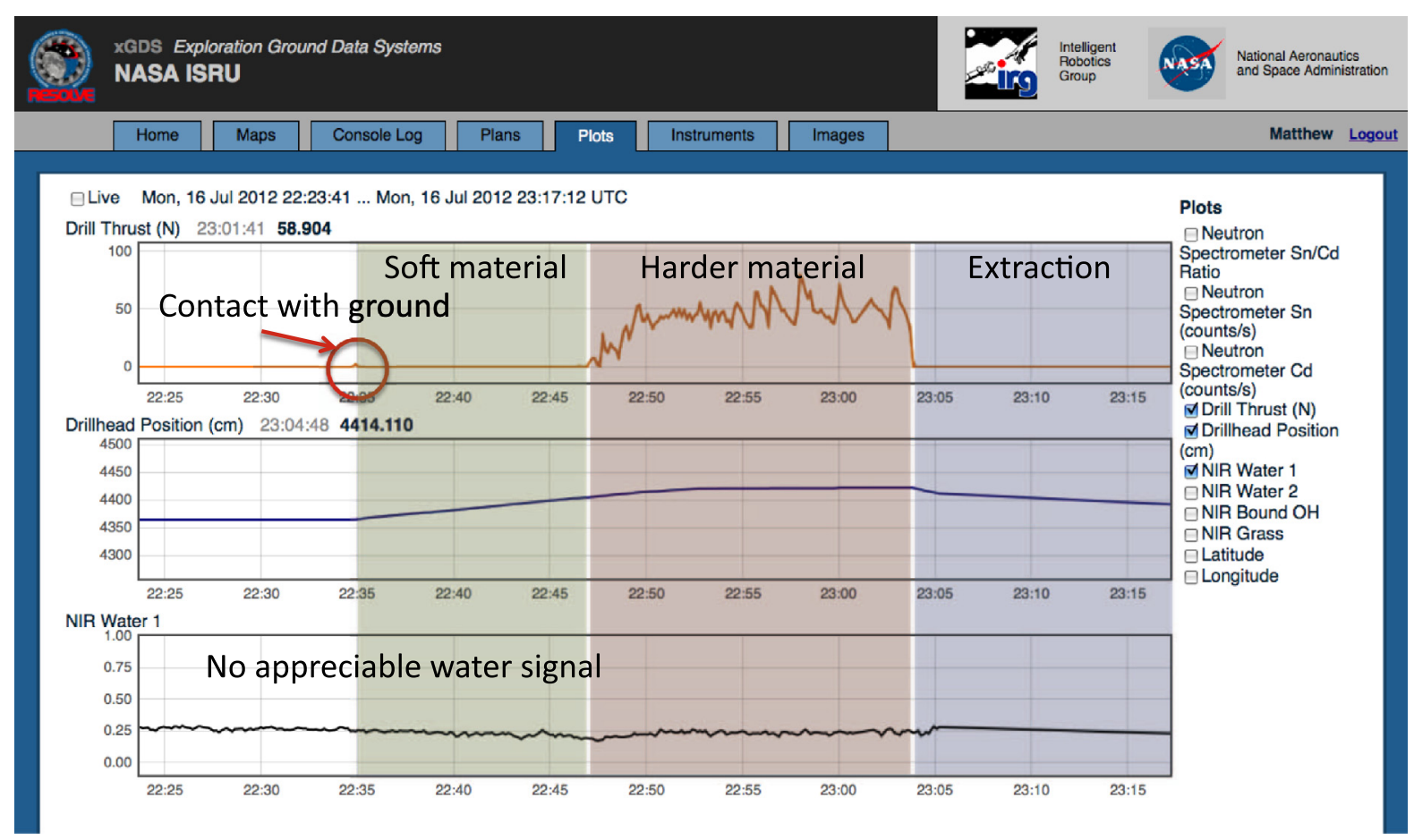

Fig. 10. Sample strip chart from the 2012 field campaign showing drill thrust (top panel), drill head position (middle panel), and near-infrared water band depth (bottom panel) as a function of time. Drill contact with the ground as well as drill penetration into soft and hard material followed by drill extraction are labeled. No appreciable water signal was detected during drill operations (bottom panel).

Science Backroom contributions included timely responses to inquiries sent down from the Flight Control Center for requests such as a verification of coordinates for drill/auger activities as recommended by the science leads in the Flight Control Center. The Science Backroom was also wellequipped to conduct more in-depth analysis of the science data. The Backroom scientists were able to follow mission operations in real-time and thus had a solid sense of science activities and datasets acquired through the xGDS quick look interfaces. The Science Backroom often took the initiative to conduct additional analyzes (such as the crosscorrelation of datasets, more detailed spectral band depth measurements, etc.) to provide a more robust understanding of the volatile distribution and trends as measured by the rover. The Science Backroom also proved proficient at preparing end of shift science reports for shift handovers and briefings for the rest of the team on science activities and findings. In general, mission operations capitalized on the Science Backroom having more science staff (compared to the Flight Control Center) to analyze science data as well as having more time since the scientists were not fully consumed with real-time data monitoring to support science decision making (as was the case for the science leads in the Flight Control Center).

\subsection{Communications}

Strict communications protocols were employed during the field campaign with several lessons learned. As previously discussed, direct communication between the Science Lead and the Flight Director was critical to ensure that science was adequately supported as a main driver of mission operations. The field test also demonstrated that the Real-Time Science Lead must be in direct communication with the rover driver(s). This direct line of communication becomes especially important when conducting science-intensive activities such as volatile hotspot localization procedures. Such procedures often require science intervention and real-time adjustments to a priori plans based on the incoming science data. The real-time nature of this mission does not allow for time delays in recommended changes to the rover driving plan if additional layers of personnel are added between the Real-Time Science Lead and the rover driver(s).

The Science Backroom also followed effective communications protocols. The Science Backroom was allowed to listen to an and all channels of communication as desired to maintain situational awareness, but was only allowed to talk directly with the Real-Time Science and Spectrometer Leads in the Flight Control Center. This configuration was designed to maintain clarity and minimize confusion for all parties. In addition, the Science Backroom had one dedicated Communications Lead for corresponding over the voice loops with the Flight Control Center science positions. These communications protocols were effective and are recommended for future field campaigns and flight opportunities. 


\subsection{Real-time decision making}

Real-time decision making based on incoming science data is the crux of operations for the lunar polar volatile rover mission. The field campaign demonstrated that the science leads must have the authority to make certain rover operations decisions without Flight Director approval. These decisions must be determined a priori (e.g., whether or not to stop driving the rover when a volatile hotspot is detected, whether or not to conduct a more intensive mapping of the hotspot location, etc). Due to the time-critical nature of this mission, there is not sufficient time to request and receive Flight Director approval through a chain of command on these types of tasks (e.g., the rover would have already driven over the area of high volatile content before the rover was commanded to stop if time was lost in requesting permission to stop). The high reliance on science positions for important mission operations decisions requires sufficient training for all mission team members to understand the rules and command responsibilities, as well as to understand mission safety and mission success rules and guidelines.

Real-time science decisions were primarily made by the Real-Time Science Lead during the Field Campaign. The field campaign demonstrated that there was generally not enough time to poll all other science positions (including the Science Backroom) for time-critical assessments and decisions. All science positions were on communication voice loops and following mission operations via xGDS, though, and so all science team members had the ability to monitor mission operations and also had the ability to dissent if a differing opinion existed on a decision. (We note, however, that there were no dissents during field campaign and all science positions felt the flight rules and guidelines were followed). The Real-Time Science Lead can (and did) ask for several minutes prior to rendering a final decision to confer with the entire science team on certain decisions (primarily reaching consensus on decisions to drill and/or auger, and getting concurrence and/or verification on coordinates for drilling).

Not all mission activities will be dictated by science, however, and certain activities (also determined a priori) will require Flight Director approval. Such activities primarily pertain to rover safety (e.g., when the rover enters into a shadowed region, etc). In such cases, engineering constraints must be considered and the Flight Director will follow a pre-determined set of checks to ensure rover safety before granting permission for the activity to occur.

\subsection{Exploration ground data systems ( $x G D S$ )}

xGDS proved to be an enabling technology during the rover field campaign. Real-time data monitoring was critical to support science decision making. The data had to be presented in an easily digestible format to enable real-time decision making by the science team (e.g., the maps and strip charts; Figs. 8-10). xGDS also archives the raw data files which proved useful particularly as Science Backroom members conducted more in-depth data analysis.

In addition to monitoring data and mission activities, xGDS also provided the ability to respond to science data in real time. For example, the science team relied on the ability to update rover traverse plans (including rover navigation and rover activities) based on incoming science data. In this manner, xGDS provided an important mechanism for two-way communications between the science team and the rover in terms of science monitoring of incoming data and the science team's ability to respond and alter the rover activity and traverse plans accordingly. xGDS also provided a method for storing and recording flight mission activities for later reference. This function was deemed important enough to assign a dedicated Stenographer Lead in the Science Backroom to ensure time-stamped notes were recorded in xGDS to document mission activities. xGDS can also display and archive time and location stamped raw data files and data products for later use and analysis by the science team. xGDS thus served as an important repository for mission critical information and data files.

An important consideration regarding the success of $x$ GDS in this field campaign is customization. Although xGDS is a generic tool that can support many different varieties of missions, certain xGDS tools were specifically tailored to support this mission. For example, the strip charts and map representations of the spectrometer datasets were specifically tailored to accept and display data from the specific rover spectrometers deployed in the field. An important component to the successful use of this software was the customization which optimized the tool for science team use.

\section{Conclusions}

The envisioned lunar mission requires highly efficient, real time, remotely operated rover operations to enable low cost, scientifically relevant exploration of the distribution and nature of lunar polar volatiles. A field demonstration conducted with a rover deployed in the lunar analog environment of Hawaii provided multiple lessons-learned to benefit future field campaigns and flight opportunities. The overall console architecture was well-designed with science interests adequately represented in the Flight Control Center and a Science Backroom providing science support and in-depth data analysis. Communications protocols were efficient and operational with a Science Lead in direct communication with the Flight Director to provide immediate and continual science support and validation throughout the mission. The Science Backroom was in voice communication with the Real-Time Science Lead and Spectrometer Lead via a Communication Lead in the Backroom but could listen to all voice channels to monitor mission activities. Real-time science decisions were the prime responsibility of the Real-Time Science Lead supported by the science team. Decisions pertaining to rover 
activities with a key safety component require Flight Director approval, and all decision-making protocols must be determined a priori. Specific data analysis tools were also required to enable immediate data monitoring, visualization, and decision making. The field campaign demonstrated that this novel methodology of real-time science operations is possible and applicable to providing important new insights regarding lunar polar volatiles for both science and exploration.

\section{Acknowledgement}

The authors thank Jerry Sanders, Bill Larson, and Jackie Quinn for their management of the July 2012 Hawaii ISRU field campaign. We thank Katie Rogers for her role in organizing the mission operations and serving as the Flight Director during the analog mission. We acknowledge the Canadian Space Agency (CSA) and Neptec for their contribution of the rover to this project, and PISCES (Pacific International Space Center for Exploration Systems) for their help in organizing the Hawaii field test.

\section{References}

Colaprete, A., Schultz, P., Heldmann, J.L., Shirley, M., Ennico, K., Hermalyn, B., Wooden, D., Marshall, W., Ricco, A., Elphic, R.C., Goldstein, D., Summy, D., Bart, G., Asphaug, E., Korycansky, D., Landis, D., Sollitt, L., 2010. Detection of water in the LCROSS ejecta plume. Science 330, 463-468.

Heldmann, J.L., Colaprete, A.C., Elphic, R., et al., 2012. RESOLVE: realtime science operations to support a lunar polar volatiles rover mission. In: LEAG meeting, Abstract \#3034, Greenbelt, MD.

Paige, D.A., Siegler, M.A., Zhang, J.A., et al., 2010. Diviner lunar radiometer observations of cold traps in the Moon's south polar regions. Science 330, 479-482.

Pieters, C., Goswami, J.N., Clark, R.N., et al., 2009. Character and spatial distribution of $\mathrm{OH} / \mathrm{H}_{2} \mathrm{O}$ on the surface of the Moon seen by $\mathrm{M}^{3}$ on Chandrayaan-1. Science 326, 568-572.

Sanders, G., Larson, W., in this issue.The importance of analog field campaigns for in situ resource utilization technology and capability maturation.

Sanders, G.B., Baird, R.S., Rogers, K.N., et al., 2012. RESOLVE lunar ice/volatile payload development and field test status. In: LEAG meeting, Abstract \#3046, Greenbelt, MD .

Sunshine, J.M., Farnham, T.L., Feaga, L.M., et al., 2009. Temporal and spatial variability of Lunar hydration as observed by the deep impact spacecraft. Science 326, 565-568. 